\title{
On Demand N-Path Routing in Wireless Networks to Improve Throughput with Congestion Control
}

\author{
P. Durgadevi \\ Research Scholar \\ Bharathiyar University \\ Coimbatore
}

\author{
N. Vetrivelan, PhD \\ Professor-Computer Application \\ Principal Srinivasan College of Arts \& Science \\ Perambalur
}

\begin{abstract}
Hybrid Wireless Network is the addition of Ad Hoc Network environment with Wireless network infrastructure. This technology is developed to provide better network experience with high performance and quality. Hybrid wireless network provides better capacity and scalability. Implementing such a network is challenging task. It needs better routing algorithm to provide flawless secured data transmission. This paper proposes, On Demand N-Path Routing, an optimized routing algorithm which will support Hybrid Wireless Network. The algorithm performs route calculation, node selection, end-toend encryption while transmitting data packets. The proposed algorithm is tested in the simulated environment which gives better results with reliability, scalability and security.
\end{abstract}

\section{Keywords}

Wireless Networking, Multipath Routing, Node Selection, Path Calculation, Routing Strategy

\section{INTRODUCTION}

The aim is to implement a high standard network environment with all supporting features. The network should be with stability, security and it should adapt topological changes. The throughput capacity of a wireless network depends on many aspects of the network. architecture of the network, energy consumption, routing technique, mobility resilient, etc. Network architecture should be designed based on its purpose and its applications [1]. Several network models are available for wireless data networks. In a normal wireless network the nodes communicate with each other via a access point or a base station. Every node in the network initially tries to connect to its nearest access point to obtain a IP for communication. A base station serves as a communication gateway for all the nodes in its cell.

Ad Hoc Network is the best solution for the dynamic environment where wireless network cannot be implemented. An Ad Hoc Network is the one which is suitable for self defined network environment as they do not need any access point to communicate [2]. Unlike normal wireless network Ad Hoc Network needs the desired routing protocol for its data transmission. Mostly Ad Hoc Network follows multi-hop transmission [2]. This paper concentrates on implementing Hybrid Wireless Network which is the combination of wireless network and Ad Hoc Network. The hybrid network is based on an ad-hoc network with an embedded wired infrastructure [3] [4]. The Hybrid Wireless Network follows both the wireless communication and node to node communication. The Hybrid Wireless Network has desired number of base stations with respect to the network size. The proposed infrastructure should adopt large infrastructure and also should provide scalability. So a defined routing algorithm should be constructed for the proposed architecture [5] [6].
The popularity of mobile network in recent days is increasing really higher. The network should not restrict the node movement over the network. There is a limitation in the number of mobile users they can simultaneously handle. Service providers are reduced their cost of service due to market competition which increased the number of service consumers [7] [8]. To suite the proposed architecture a better algorithm has to be implementing to provide better throughput. The throughput of the Hybrid Wireless Network can be calculated by obtaining transmission rate between the random send to the random receiver [9]. When the sender node sends data to the receiver node all other nodes in the network act as the route node to transmit data [10].

So a network by the combination of wireless network and Ad Hoc Network can be implemented in the name of Hybrid Wireless Network. The challenging thing is the implementing of network in large area networks. The network environment should have the capacity to switch between transmission types from wireless to Ad Hoc transmission [11] and the effective utilization of the nodes in the network. The data transmission should not affect the lifetime of the node in the network by draining the battery power [15] [16]. It is the purpose of this work to study the capacity of Hybrid Wireless Network. In implementation of this network the following points are important.

- Calculating the throughput capacities between nodes and base-stations.

- Managing the size of the network with respect to new connections.

This paper proposes a hybrid network model with On Demand N-Path Routing to improve network connectivity and the Stability. In the proposed model the base station is connected with the wireless node which also a member of Ad Hoc Network. The proposed network consists of normal nodes and some well-connected base stations. It is called hybrid network as it following the Ad Hoc Properties even in wireless network. The Ad Hoc Network has no infrastructure and follows multi-hop transmission. In cellular network data packets are transmitted between one another. But in Hybrid Wireless Network the data can be transmitted in wireless fashion or by Ad Hoc Fashion. Communication path for mobile communication and data communication is differed in our proposed model. It will be presented, simulated and result is analyzed with the proposed model.

\section{RELATED WORK}

To continue our proposed work some survey is made on routing and environmental setup of most of the Ad Hoc Network. Many approaches have proved that node to node communication in Ad Hoc Networking gives better throughput. But in the same way the properties of the Ad Hoc Network should not be altered in Hybrid Wireless Network 
[19]. Some papers proved that the possibility of implementing Ad Hoc Network in wireless network. This paper also proves that the Ad Hoc Network is also mobility resilient network. But some problems should be faced while using Ad Hoc Network it is prone to energy loss so a better algorithm hat to be implemented to manage energy efficiency. A new distributed routing protocol for mobile, multi-hop, wireless networks can be implemented. This algorithm concentrates on diffusion of data via nodes in link reversal manner to reduce data loss. The protocol is proved to be suitable for large network environment. To overcome link failure this algorithm uses the single pass manner. The protocol should be stable, adaptive to any network environment. They refer to the protocol as the Temporally-Ordered Routing Algorithm (TORA) [19]. The purpose of this paper is to analyze the routing algorithms that how support full with wireless network. The comparison is done by varying the number of nodes periodically in the network. A new network environment with nodes under particular base station is with desired space can be provided where the nodes will follow the Ad Hoc manner. Some research shows that bottleneck problems may occurs at Multi-Hop Transmission. Results obtained on actual throughput of data confirm our findings. In Ad Hoc network the node act as router based on its routing situation. In such environment the node may not able to transmit data or may fails due to power loss. This paper presents a protocol for routing in ad hoc networks that uses Ad Hoc Nodes to include in the routing table [20]. The protocol will be adoptable to mobility resilient. The algorithm will be suitable to any Hybrid Wireless Network Environment with any change. Some of the previous approaches made successful performance with Single Hop transmission but faced transmission failures and delay transmission with multihop transmission.

\section{PROBLEM STATEMENT}

The possible problems are stated below that should be faced while implementing the Hybrid Wireless Network,

- The network should be adaptive to continuously changing size of the network.

- The data communication should not spoil the wireless mobile communication.

- The data communication should be performed in secured fashion.

- All Possible communication paths should be calculated for multi-hop communication.

- Selection of node for path calculation should be done with care.

\section{PROPOSED MODEL}

\subsection{On Demand N-Path Routing}

To provide better network throughput, security traffic avoidance this paper proposes On Demand N-Path Routing. This algorithm could be implemented on Hybrid wireless network which can perform the following action. Unlike the existing system On Demand N-Path Routing will not divide the data into several segments. It will first calculate available routes via available base stations. Then based on the paths available on the network the data is divided into N Segments. Then the sender will made $\mathrm{N}$ Threads to transmit data to $\mathrm{N}$ base stations and waits for positive feedback. The second part of the algorithm which runs on the Base Station will has the responsible to avoid congestion and traffic on the network and also to choose proper node for data transmission.

\subsection{Hybrid Wireless Network Setup}

The proposed wireless network architecture is in Hybrid fashion. The network will consist of base stations and mobile nodes. While the nodes connected to the base-station to form wireless network the node themselves connected together to form Ad Hoc Network. The wireless network which is based on base station is with high performance and more reliable one. And also the Ad Hoc network is more flexible and adaptive. The base station network is taken for the mobile communication where the Ad Hoc network is utilized for mobile data communication. This paper mainly concentrates on mobile data communication by not disturbing mobile communication. The following diagram shows our proposed architecture from Hybrid Wireless Network environment. This network environment will be implemented in NS2 simulator for experimental implementation which is shown in Fig 1. Proposed Hybrid Wireless Network.

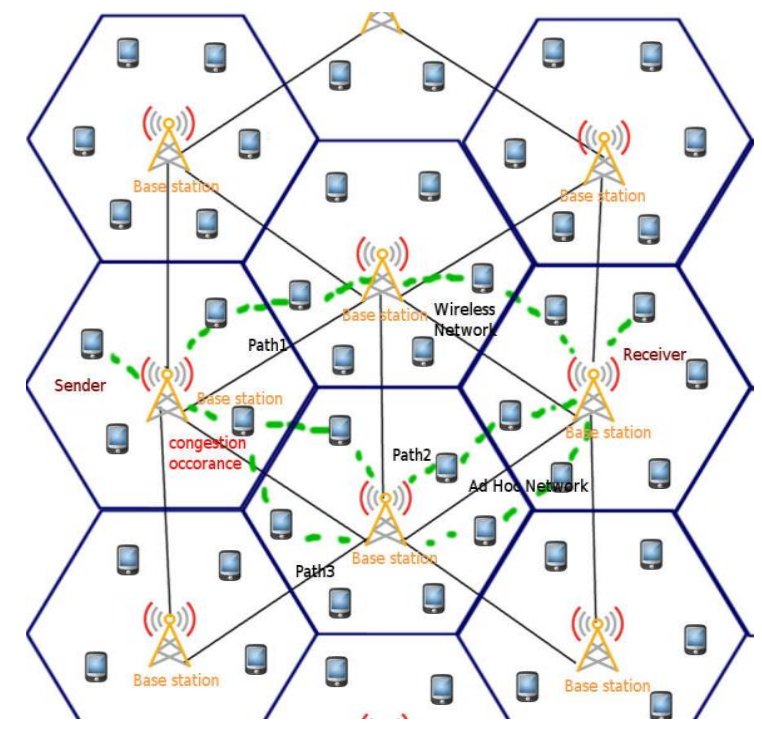

Fig 1. Hybrid Wireless Network Setup

\subsection{Base Station Selection}

The first step of transmitting data from source to destination, routing path must be calculated. To calculate the routing path base station and nodes are needed. As the initial step, the node in the network should connect to the nearest base-station. To find the nearest base station the source node will send some ping signals. The ping signals will be received by any one base-station. As a reply the base-station will send back the ping response to the source node. By this way the source node can find the nearest base station. Then the node will connect to the base-station by obtaining unique IP. Then the source node sends all its data to the base-station after encrypting it with the receiver nodes ID.

\section{Algorithm 1. Base Station Selection}

\section{start}

while ping

send response

get response

if response and retrieval

get response time

sort response time

end if

select base station from list

end 


\subsection{Secure Node Selection}

Node selection is one of the most important processes in the routing path calculation. Before calculating routing path from source to the destination, the best nodes that have the capabilities to transmit data packets should be identified. The capabilities include node stability, mobility, energy consumption, buffer capacity and traffic on node should take in account. So the base-station will select those types of nodes for routing.

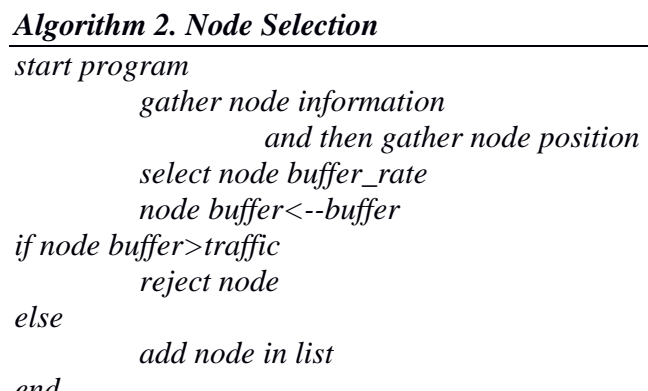

\subsection{N-Path Route Calculation}

This step of the algorithm involves in routing path calculation from the source node to the destination node. The routing calculation is done by the base-station which holding the source node. Let base-station where the source node held as source base-station. The source base-station sent the request to the neighbor base-stations for identifying the location of destination node. The source base-station will receive the response from neighbor base-stations. Using this response message, the base-station with destination node will be identified, say it as destination base-station. Now the routing path has to be calculated from source base-station to destination base-station. But the communication will not occur between base-station to base-stations. The algorithm is going to use intermediate nodes between the base stations for routing path calculation. The path will derive using basestation to intermediate node and intermediate node to basestation.

\subsection{Data Segmentation}

The data segmentation is done for the purpose to transmitting the data much faster in multiple paths. The segments count is equal to the number of available path on the network. The data is divided into several paths and submitted to the available base station on the networks. Then it is responsible of the base station to send the data to the receiver nodes.

\begin{tabular}{cl} 
Algorithm 3. Data segmentation \\
\hline start & get data \\
& convert data into byte array \\
& get count $n<--n o$ of base station \\
& segment data into $n$ parts \\
& create transmission thread \\
& send data \\
end data &
\end{tabular}

\subsection{Transmission Thread Creation}

It is to create network connections and communicate through them, the fact that network communication is asynchronous. A connection thread along with the transmission thread will be created on the base-station. Then it will convert the data into UDP packets and then transmitted to available nodes on the wireless network. Then the nodes in the network which also forms a Ad Hoc Network by themselves forwards that data packets to the available neighbors in AODV scheme to the destination base-station. This process will be continued till all the data packets transmitted to the destination base-station. Finally it is responsible of the destination base-station to send the data to the destination node.

\subsection{Route Overlap Monitoring}

Its aim is to detect and monitor the route overlapping in the network to avoid congestion. Congestion in network occurs when a node involves more than one data transmission at a time. If congestion occurs on the network its qualities gets reduced. Throughput, stability and robustness everything will be gone worst. It is responsibility of part of the algorithm to monitor on congestion occurrence on the network. So the algorithm is instructed to monitor on paths which is calculated for data transmission from base-station to base-station. If a node seems to be present on more than one routing path then the congestion controller will apply scheduling algorithm to avoid data loss due to congested transmission.

\section{Algorithm 4. Overlap Monitoring}

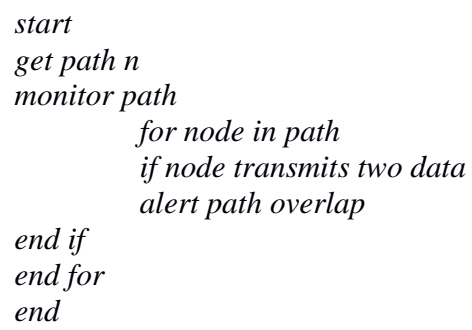

\section{EXPERIMENTAL EVALUATION 5.1 Experimental Setup}

The proposed network architecture is implemented and tested on NS2 simulated environment. The NS2 version is 2.35 is installed in Unbent 14.04 with 4 GB of RAM memory. The HWN is implemented in NS2 with various counts of basestations and network nodes. The nodes are allowed to move randomly thus the network will be dynamic. The nodes can connect to any base-station and communicate via that network. The proposed architecture shows better result and performance. The result gives better throughput, less traffic, lower congestion rate and lower data losses.

\subsection{Experiment}

The NS2 simulated is implemented with the various number of node counts. The time bound is set to 1 minute. The nodes are allowed to form as a cluster under a base station. Some to the nodes is implementing to act as the base-station. The nodes near to the base-station are allowed to connect to it for further communication. Some of the nodes made to act as the sender nodes allowed to send 10MB of data to the desired receivers where some nodes allowed acting as the receiver nodes. Every transmission occurs for various time period based on the distance between the sender and the receiver lies on the topography. Transmission rate is recorded for every single transmission in time interval of seconds. Using this transmission rate throughput, congestion and packet drops can be calculated.

\subsection{Parameters Used}

1.Throughput $=$ File Size $/$ Transmission Time $($ bps $)$

The above formula is used to calculate the throughput of the transmission. The calculation is done by identifying the ratio between file size and the transmission time. 


\section{Packetloss=No. of packet Sent-Ack Recieved}

Packet loss is calculated by finding the difference between the number of packet sent and acknowledgement received for specific amount of transmission.

\section{CongestionRate=TrafficOnNode/Buffer Size}

Congestion rate is calculated by dividing the Traffic rate on nodes by the buffer size of that particular node under traffic.

The following graph shows the performance chart with respect to number of nodes. The experimental evaluation result is showed in the following table 1 .

\begin{tabular}{|l|l|l|l|}
$\begin{array}{l}\text { No. of } \\
\text { nodes }\end{array}$ & $\begin{array}{l}\text { Throughput } \\
\text { (KBPS) }\end{array}$ & $\begin{array}{l}\text { Congestion } \\
\text { Rate(KBPs) }\end{array}$ & $\begin{array}{l}\text { Packet } \\
\text { Loss } \\
\text { (KBPS) }\end{array}$ \\
\hline 30 & 56 & 20 & 16 \\
\hline 60 & 57 & 22 & 15 \\
\hline 80 & 65 & 21 & 18 \\
\hline 100 & 67 & 27 & 17 \\
\hline
\end{tabular}

The below shown graph shows that the amount of throughput, Congestion and Packet loss for the various given amount of nodes.

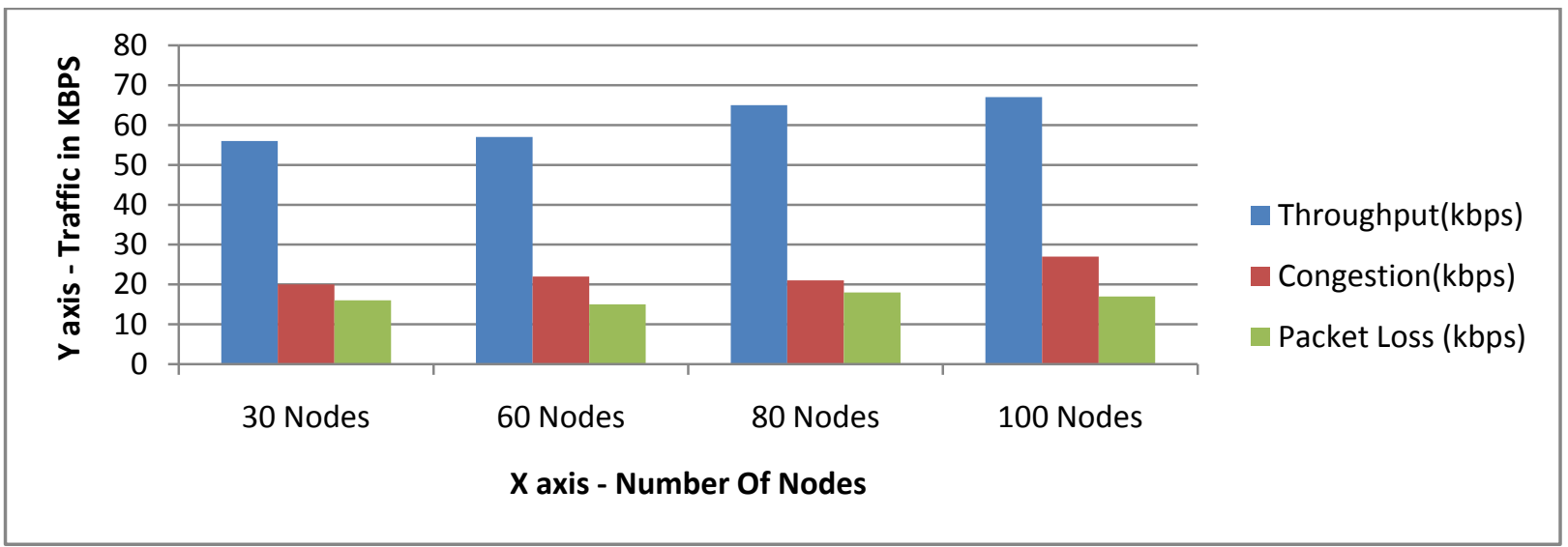

Chart 1. Node vs. Throughput, Congestion and Data loss

\section{CONCLUSION}

This paper revealed the throughput capacity of hybrid wireless networks. A hybrid network consists of an ad hoc network and a sparse network of base stations. The base stations are connected by a wired network and placed in the ad hoc network in a regular pattern. Data may be forwarded in a multi-hop fashion as in ad hoc networks or forwarded through the infrastructure as in cellular networks. The work has been done to investigate the benefit of the infrastructure to the throughput capacity and derive the asymptotic capacity of hybrid networks.

\section{FUTURE WORK}

Hybrid Wireless Network will be a well suited network for the present technology. But it is doubtful that will it may support the future networking environments. The AODV method can be optimized fully to support Ad Hoc scheme of routing. Some time delay occurs in Ad Hoc routing scheme it should be overcome at future. The concentration only made on the mobile data communication at present. In future the mobile communication has to be improved in the mobile communication in Hybrid Wireless Network.

\section{REFERENCES}

[1] Khondaker M. Salehin and Roberto Rojas-Cessa, "Active Scheme to Measure Throughput of Wireless Access Link in Hybrid Wired-Wireless Network", IEEE Wireless Communications Letters, VOL. 1, NO. 6, DECEMBER 2012.

[2] M. d. P. Salamanca, N. M. Peña and N. L. S. da Fonseca, "A Distributed Envelope-Based Admission Control for Multihop IEEE 802.11 Ad Hoc Networks", IEEE Latin America Transactions, Vol. 11, No. 3, May 2013.

[3] Haiying Shen, Senior Member, IEEE, Ze Li, and Chenxi
Qiu, "A Distributed Three-Hop Routing Protocol to Increase the Capacity of Hybrid Wireless Networks", IEEE Transactions On Mobile Computing,Vol. 14,No. 10, October 2015.

[4] Fan Wu, Member, IEEE, Sheng Zhong, Member, IEEE, and Jiqiang Liu, Member, IEEE, “An Optimal, StrategyProof Scheme for Multi-Path Traffic Assignment in NonCooperative Networks", IEEE Transactions On Wireless Communications, Vol. 9, No. 3, March 2010.

[5] Yi Song, Jiang Xie, Senior Member, IEEE, and Xudong Wang, "A Novel Unified Analytical Model for Broadcast Protocols in Multi-Hop Cognitive Radio Ad Hoc Networks", IEEE Transactions On Mobile Computing, Vol. 13, No. 8, August 2014.

[6] Haiying Shen, Senior Member, IEEE, Ze Li, Student Member, IEEE, and Lei Yu, "A P2P-Based MarketGuided Distributed Routing Mechanism for HighThroughput Hybrid Wireless Networks", IEEE Transactions On Mobile Computing, Vol. 14, No. 2, February 2015.

[7] Weihuang Fu, Member, IEEE, and Dharma P. Agrawal, Fellow, IEEE, "Capacity of Hybrid Wireless Mesh Networks with Random Aps",IEEE Transactions On Mobile Computing, Vol. 12, No. 1, January 2013.

[8] Guanglin Zhang, Youyun Xu, Member, IEEE, Xinbing Wang, Member, IEEE, and Mohsen Guizani, Fellow, IEEE, "Capacity of Hybrid Wireless Networks with Directional Antenna and Delay Constraint", IEEE Transactions On Communications, Vol. 58, No. 7, July 2010.

[9] Khajonpong Akkarajitsakul, Member, IEEE, Ekram 
Hossain, Senior Member, IEEE, and Dusit Niyato, Member, IEEE, "Cooperative Packet Delivery in Hybrid Wireless Mobile Networks. A Coalitional Game Approach", IEEE Transactions On Mobile Computing, Vol. 12, No. 5, May 2013.

[10] Shiva Murthy G, Robert John D'Souza, and Golla Varaprasad, "Digital Signature-Based Secure Node Disjoint Multipath Routing Protocol for Wireless Sensor Networks", IEEE Sensors Journal, Vol. 12, No. 10, October 2012.

[11] Bin Wang, Dongmei Zhao, and Jun Cai, "Joint Connection Admission Control and Packet Scheduling in a Cognitive Radio Network with Spectrum Underlay", IEEE Transactions On Wireless Communications, Vol. 10, No. 11, November 2011.

[12] Cheng Wang, Student Member, IEEE, Xiang-Yang Li, Senior Member, IEEE, Changjun Jiang, Member, IEEE, Shaojie Tang, Student Member, IEEE, and Yunhao Liu, Senior Member, IEEE, "Multicast Throughput for Hybrid Wireless Networks under Gaussian Channel Model", IEEE Transactions On Mobile Computing, Vol. 10, No. 6, June 2011.

[13] Saiful Azad, Paolo Casari, Senior Member, IEEE, and Michele Zorzi, Fellow, IEEE. "Multipath Routing With Limited Cross-Path Interference in Underwater Networks", IEEE Wireless Communications Letters, Vol.

\section{3, No. 5, October 2014.}

[14] Xin Wang, Student Member, IEEE, and Qilian Liang, Senior Member, IEEE, "On the Throughput Capacity and Performance Analysis of Hybrid Wireless Networks over Fading Channels", IEEE Transactions On Wireless Communications, Vol. 12, No. 6, June 2013.

[15] Amlan Ganguly, Member, IEEE, Kevin Chang, Student Member, IEEE, Sujay Deb, Student Member, IEEE, Partha Pratim Pande, Member, IEEE, Benjamin Belzer, Member, IEEE, and Christof Teuscher, Member, IEEE, "Scalable Hybrid Wireless Network-on-Chip Architectures for Multicore Systems", IEEE TRANSACTIONS ON COMPUTERS, VOL. 60, NO. 10, OCTOBER 2011.

[16] Erik Kuiper and Simin Nadjm-Tehrani, Member, IEEE,"Geographical Routing With Location Service in Intermittently Connected MANETs", IEEE Transactions On Vehicular Technology, Vol. 60, No. 2, February 2011.

[17] Ziming Zhao, Student Member, IEEE, Hongxin Hu, Student Member, IEEE, Gail-Joon Ahn, Senior Member, IEEE, and Ruoyu Wu, Student Member, IEEE,"RiskAware Mitigation for MANET Routing Attacks", IEEE Transactions On Dependable And Secure Computing, Vol. 9, No. 2, March/April 2012. 